\title{
Übersetzer und der technische Fachtext. Über das Los eines Sprachmittlers oder ein Blick in sein Arbeitszimmer
}

\author{
Olga Rösch
}

\section{Vorbemerkung}

Wissenschaftliche Fachliteratur wird sowohl in Deutschland als auch in anderen nicht-anglophonen Ländern zunehmend in Englisch verfasst. Dieser Trend ist insbesondere für die Veröffentlichungen in den naturwissenschaftlichen Disziplinen kennzeichnend. Die zunehmende Internationalisierung, und insbesondere die Vernetzung von jeweiliger nationaler Wissenschaft und Wirtschaft, bedeutet aber für Europa nicht automatisch eine Umstellung aufs Englische. Denn Internationalisierung ist ein tiefergehender mentaler Prozess der Öffnung ${ }^{1}$, der sich auf der „Oberfläche“ u.a. auch in der Nutzung der Lingua Franca Englisch manifestieren kann, es aber nicht muss.

So besteht ein großer Bedarf an Übersetzungsleistungen auch aus dem und in das Englische. Dies geht aus den neueren statistischen Erhebungen zur Analyse des Arbeitsmarktes für Sprachmittler hervor (vgl. Schmitt, P., 1998). Selbst in der Wirtschaft, wo sich das Englische im Bereich der Auslandskontakte etabliert hat, ist die Nachfrage nach Übersetzungen erheblich. Rund $80 \%$ der befragten deutschen Unternehmen benötigen die Leistungen der Sprachmittler. Bei einer Aufschluisselung des Translationsbedarfs nach Sprachen ergibt sich in Deutschland die Reihenfolge: Englisch, Französisch, Spanisch, Italienisch, Russisch, Portugiesisch - als wichtigste sechs Sprachen in die bzw. aus denen ins Deutsche übersetzt wird (vgl. ebd. S. 5ff.). Differenziert man den Bedarf nach Branchen, so entfällt ein Drittel des Translationsvolumens in Deutschland auf den facettenreichen Themenbereich Metallbau/ Maschinenbau/ Kfz-Technik. Ein weiteres wichtiges Fachgebiet ist E-Technik/ EDV/ Telekommunikation. In diesem Themenbereich beträgt das Auftragsvolumen für Übersetzungsleistungen z.B. bei Siemens-Nixdorf (SNI) ca.10 Mio. DM pro Jahr (vgl. ebd.).

Die Übersetzung eines naturwissenschaftlichen oder eines technischen Textes bedeutet für einen Philologen, der in der Regel Sprachmittler ist, zumeist „Weiterbildung“ auf dem jeweiligen neuen Fachgebiet, also eine zusätzliche Investition, die neben der eigentlichen $\mathrm{Ar}$ beit - der Übersetzung (im engeren Sinne) des Textes aus der Ausgangs- in die Zielsprache - erfolgt. Wissen wir doch, dass sich eine Übersetzung nicht in der Summe der korrekt übersetzten Wörter erschöpft.

Aber wieviel (Fach-)Kompetenz traut man einem Übersetzer von Fachtexten iiberhaupt zu?
Während die Arbeit eines Wissenschaftlers z.B. an seinen veröffentlichten Forschungsergebnissen gemessen werden kann, bleiben für einen Außenstehenden die Leistungen eines Sprachmittlers weitgehend verborgen, es sei denn, der letztere hat die im Ausgangstext positiv hervorgehobenen Forschungsergebnisse in seiner Übersetzung negativ dargestellt. Von entsprechenden Konsequenzen für den Übersetzer kann ausgegangen werden.

In meinem Beitrag möchte ich einige Fragen der Übersetzung von Fachtexten aus der Sicht des Praktikers beleuchten. Dabei werde ich mich hier vorwiegend auf diejenigen Arbeitsschritte konzentrieren, die die Produktion eines Translats - eines Fachtextes in der Fremdsprache - begleiten. Im Anschluss werde ich auf einige Fragen der Übersetzung einer Bedienungsanleitung aus dem Deutschen ins Russische eingehen.

\section{1. Übersetzer und Fachübersetzung}

\subsection{Der Markt}

Stellen wir uns die Situation eines Sprachmittlers, und zwar, eines Absolventen vor, der nach seinem Studium auf der Arbeitsuche ist. Eine unbefristete Stelle in einem Bundespatentamt ist zunächst nicht in Sicht (z.B. mangels einschlägiger Berufserfahrung o.ä.). Den Traum auf Regierungsebene zu dolmetschen oder einen neuen Roman aus der Feder eines Georg-Büchner- oder gar eines Nobelpreisträgers zu übersetzen - den Traum hat man noch nicht ausgeträumt, aber erst einmal in die Zukunft „verlegt". Vernuinftigerweise widmet man sich dem akuten Problem der Existenzsicherung.

Es gibt natürlich mehrere Tätigkeitsfelder fuir einen Sprachmittler, z.B. als Fremdsprachenkorrespondent, technischer Redakteur, oder indem man ein Übersetzungsbüro gründet bzw. sich einem solchen anschließt. Eine der Möglichkeiten bliebe immer offen - sich selbständig zu machen und nach Aufträgen zu suchen. Für einen Berufsanfänger ist dies keine einfache Situation. Lässt man sich beeidigen, dann bekommt man die Chance, bei Gerichten zu dolmetschen und Urkunden der verschiedensten Art zu übersetzen.

Mit welcher Art von Aufträgen hat man als Sprachmittler überhaupt zu rechnen? Dieser Frage ist P. Schmitt (1988) in seiner o.g. Untersuchung zu Marktsituation der Über- 
setzer nachgegangen. Die Erhebungen zur Häufigkeit der Textsorten in der Translationspraxis haben ergeben, dass an erster Stelle die Geschäftskorrespondenz steht. Ihr folgen Benutzerinformationen, also Anleitungen und Anweisungen vielfältiger Art, die für Übersetzungsbuiros sogar die mit großem Abstand wichtigste Textsorte sind.

Nun hat man endlich einen Übersetzungsauftrag: es ist ein technischer Fachtext, der für eine Fachzeitschrift bestimmt ist, z.B. zur Thematik: „Stufenwirkungsgrad und Extraktionsmittelverbrauch in der Flüssig-Extraktion: Vergleich Mittler-Settler und pulsierter Siebboden in der Miniplanttechnik". ${ }^{2}$ Zunächst ist man natürlich froh, dass man eine Arbeit bekommt, die auch bezahlt wird. Dann schaut man sich den Originaltext in Deutsch genauer an.

Was empfindet wohl ein Sprachmittler beim ersten Lesen eines solchen Textes aus einem unbekannten Fachgebiet? Als Übersetzer müssen wir immer wieder feststellen, dass das Allgemeinwissen nicht ausreicht, um einen Fachtext auf Anhieb zu verstehen, und dies weder inhaltlich noch sprachlich, obwohl er in der Muttersprache vorliegt. Wie soll man den Text nur in die Fremdsprache übertragen? Man kommt sich irgendwie sprachlich inkompetent vor.

\subsection{Die Muttersprache}

Zu einer realistischen Einschätzung der eigenen Sprachkompetenz verhilft uns Hans Hönig (1988) in seinem Buch „Konstruktives Übersetzen“, wenn er schreibt: „Niemand ist hundertprozentig sprachlich kompetent - nicht nur in der Fremdsprache nicht, sondern auch nicht in der Muttersprache. Die ,deutsche Sprache ist ein Sammelbegriff für eine große Anzahl von Sprachvarianten (wie z.B. Fachsprachen, Soziolekte, Dialekte), die kein kompetenter Verwender der deutschen Sprache gleichermaßen verstehen und verwenden kann."

Dann stellt er eine wohltuende rhetorische Frage:

„Wer von uns könnte einen juristischen Schriftsatz abfassen (oder auch nur verstehen), eine technische Beschreibung formulieren, alle regionalen Varianten der Umgangssprache verstehen? Das heißt: Wir sind - auch in unserer Muttersprache nur relativ kompetent." (s. Hönig, 1988:55).

So beruhigend diese Worte auch sein mögen, bedeuten sie für den Übersetzer immerhin, dass wir uns die fehlende sprachliche Kompetenz auf einem bestimmten Fachgebiet in einer relativ kurzen Zeit erarbeiten müssen.

Als Philologe kommt man in der Regel weder wegen des Inhalts noch wegen sprachlicher Raffinessen eines technischen Fachtextes ins Schwärmen. Also, man geht nüichtern an die Arbeit und beginnt den Text unter dem Gesichtspunkt der Übersetzbarkeit (auf zunächst mikrostruktureller Ebene, d.h. Wortverbindungen, Termini) zu studieren. Dabei muss man meistens feststellen, dass schon der Ausgangstext sprachlich zu wünschen übrig lässt. So sei hier als Beispiel eine grammatisch zwar korrekte, aber nicht gerade leserfreundliche Textpassage aus der Fachzeitschrift „Wissenschaftliche Beiträge. Forschung, Lehre, Technologietransfer" der TFH Wildau angeführt:
„... Im Blick auf eine Kristallisation unter verarbeitungsnahen Bedingungen, ohne Beeinflussung durch den Meßvorgang, die zudem den zu messenden Geschwindigkeitsbereich abdecken kann, schränkt sich die Zahl der Methoden und der auswertbaren Größen stark ein. ..."3

Oder eine grüblerisch stimmende Kapitelüberschrift aus unveröffentlichten Lehrmaterialien:

„Empfehlungen zum Handlungsbedarf...“.

Lässt sich denn der Bedarf überhaupt empfehlen?...

Im nachhinein, wenn man es dennoch geschafft hat, das in die Fremdsprache zu übertragen, was der Autor damit gemeint haben könnte, hebt man für sich eher die positive Wirkung einer solchen Formulierung hervor: Hat sie doch einen beim Übersetzen zum Nachdenken iiber die deutsche Sprache veranlasst! Eines steht aber fest: Je schwieriger der Originaltext, um so besser sehen für einen Sprachmittler die Chancen aus, den Übersetzungsauftrag zu bekommen.

Als Berufsanfänger ärgert man sich noch besonders über die sprachliche Unvollkommenheit der Originaltexte. Trost findet sich abermals bei H. Hönig (1988) im oben erwähnten Buch. Er schreibt:

„Die überwiegende Mehrzahl der Texte, die übersetzt werden, ist von Laientextern hergestellt. Laientexter sind Menschen, die nicht deshalb das Wort ergreifen, weil sie besonders gut oder reflektiert damit umgehen können, sondern weil sie aufgrund ihrer beruflichen Qualifikation oder ihrer Stellung in der Gesellschaft etwas zu sagen haben. “ (Hönig, 1995:96).

Und an einer anderen Stelle äußert er sich über die Beschaffenheit der Originaltexte:

"Jeder Text hat Defekte, wenn wir normative oder absolute Maßstäbe anlegen, aber andererseits können wir mit gleichem Recht feststellen: So gut wie alle Texte funktionieren - insofern haben sie auch keine Defekte.“ (ebd., S. 137).

Der Übersetzer kann sich jedoch nicht leisten, sich hinter dem unvollkommenen Original zu verstecken. Es wäre sicherlich auch realitätsfremd zu erwarten, dass die technischen Fachtexte primär unter sprachpflegerischem Aspekt verfasst würden. Die erfahrenen Sprachmittler können nur bestätigen, dass auch verschrobene Texte ihre Funktion unter den Fachleuten erfuillen, da sie offensichtlich verständliche Inhalte transportieren, und kaum einer nimmt Anstoß an der sprachlichen Unzulänglichkeit der Originaltexte (ich betone: Originaltexte! An den übersetzten Text wird oft ein anderer Maßstab angelegt!). Dem Auftraggeber ist ein Produkt der Translation abzuliefern, das sprachlich korrekt sowie sachlich richtig und für die zuvor präzise definierte Gruppe von Adressaten funktionsfähig ist.

\subsection{Die Hilfsmittel}

Wie geht man nun vor, wenn der Text in einer Sprachvarietät, nämlich Fachsprache, verfasst ist, die nur am Rande der eigenen Sprachkompetenz liegt, und die Wissensbestände (Sachwissen) nicht ausreichen, um den Text zu verstehen? Der Fachübersetzer beginnt mit 
der Erarbeitung von Makrostrategien (vgl. Hönig, 1995:55f.) und fragt sich zunächst: Durch welche Recherchen kann ich meine Sachkompetenz erweitern?

Der kürzeste und günstigste Weg, sich kundig zu machen, ist eine Konsultation mit dem Fachmann. Im Idealfall (sprich: im Gliicksfall!) nimmt sich der Fachmann Zeit, den Text zu iiberfliegen, falls er nicht von ihm stammt, weiht den Übersetzer in das Thema ein, umschreibt auf Deutsch, worum es geht, entziffert die unauffindbaren Abkürzungen, erklärt die für das Verstehen wichtigsten Termini, empfiehlt ein Fachlexikon und drückt dem Wissensdurstigen sogar ein paar Paralleltexte $^{4}$ in die Hand. Im Normalfall kommt es jedoch eher anders. Der Fachmann sagt dem ratsuchenden Sprachmittler herablassend-mitleidig: „Das brauchen Sie doch nicht zu verstehen, Sie müssen ja nur übersetzen!"

Nun bleibt der Übersetzer mit dem Text auf sich angewiesen. Es ist ratsam, mit der Aufstellung einer Terminologieliste zu beginnen, in der die im Text vorgekommenen Begriffe definiert sind. Je nach Fachgebiet kann die Terminologiearbeit unterschiedlich aufwendig sein. Es gibt auch Fachbegriffe, deren allgemeinsprachliche Bedeutung aus dem Kontext erschließbar ist. Wenn man z.B. einen Fachtext aus der Landwirtschaft liest und den Terminus „rauhfutterverzehrende Großvieheinheit" findet, so wird man schon erraten, dass es um das Hausrind, also meist die Kuh geht.

Für die Erarbeitung der Terminologieliste benötigt der Sprachmittler oft mehrere Nachschlagewerke: einsprachige Sachwörterbücher, Lexika bzw. Enzyklopädien und auch Paralleltexte und Hintergrundtexte ${ }^{5}$. Die Aufstellung einer Terminologieliste in der Ausgangssprache gehört noch zu den Verstehensstrategien bei der Vorbereitung einer Fachübersetzung. Erst im nächsten Schritt geht der Fachübersetzer zur Wiedergabe des Originaltextes in der Fremdsprache über. Hier können die Schwierigkeiten bereits bei der Suche nach guten zweisprachigen Fachwörterbüchern beginnen. Wenn es ums Russische geht, so finden sich einige Hinweise in der „Bibliographie zur russischen Fachsprache. Forum für Fachsprachenforschung“" von Birkenmaer/Mohl (1990).

Die Zuverlässigkeit der zweisprachigen Wörterbücher, jedenfalls was das Sprachenpaar Deutsch-Russisch betrifft, ist ein leidiges Thema. Viele Wörterbücher sind schlicht veraltet. Bei den neu erschienenen handelt es sich nicht immer um die neu ïberarbeiteten Auflagen. Die tatsächlich neu zusammengestellten Wörterbüicher - meist geht es um die fachliche Sprachwörterbücher Wirtschaft, Finanzwesen u.ä. - haben, was die grammatisch-lexikalischen Einträge, Explikationen, Definitionen und sonstige Systematik betrifft, oft nicht das erforderliche Niveau. Manchmal bieten sie auch einfach falsche Übersetzungen an.

Nehmen wir hier als Beispiel das im Cornelsen-Verlag (1993) erschienene deutsch-russische „Wörterbuch Wirtschaftsrussisch" von Anna Nikiforowa. Dort wird das Wort "Ökosystem“ als „экономическая система" (= Wirt- schaftssystem) ïbersetzt (vgl. S. 293). Eine ähnliche Kuriosität findet sich im „Polytechnischen Wörterbuch" von Alexander Pankin, Moskau (1995). In dem Wörterbuch wird für den Fachausdruck ,benutzerfreundliche Schnittstelle" die Übersetzung „дружественный интерфейс" (= freundschaftliche Schnittstelle) angeboten (vgl. S. 502).

Die Erfahrung mit der Unzuverlässigkeit der zweisprachigen Wörterbücher macht sicherlich jeder Übersetzer. Da auf sie dennoch nicht verzichtet werden kann, empfiehlt es sich, gleichzeitig mehrere Fachwörterbuicher zu konsultieren, und zwar, in unserem Falle deutsch-russische, die in Russland in verschiedenen Verlagen erschienen sind und von verschiedenen Autoren verfasst wurden sowie russisch-deutsche, die in Deutschland zusammengestellt und ebenfalls von verschiedenen Verlagen und Autoren herausgegeben worden sind. Sehr hilfreich können auch mehrsprachige Wörterbuicher sein. So z.B. das deutsch-englisch-russische „Enzyklopädische Wörterbuch: Markwirtschaft und Planwirtschaft" (1992) in 2 Bänden, oder das fünfsprachige „Fachwörterbuch der Mikroelektronik“, PONS, (1991), u.a..

Unentbehrlich sind aber vor allem die einsprachigen Nachschlagewerke in der Zielsprache: fachliche Sachwörterbuicher, Lexika und Enzyklopädien. Für die Übersetzung ins Russische als Fremdsprache, ist es sinnvoll auch „Словарь сочетаемости слов русского языка“ von Denissow P.N u.a. (1983) zu konsultieren, um die semantische Kompatibilität einiger Wortverbindungen im eigenen Text (bei Bedarf versteht sich!) zu uiberprüfen.

Wenn Deutsch die Fremdsprache ist, in die der Fachtext uibersetzt werden muss, so kann „Das neue einsprachige Wörterbuch für Deutschlernende" (1997) sehr hilfreich sein, in dem die Einträge mit sämtlichen morphologischen, grammatisch-lexikalischen und orthographischen Charakteristiken versehen sind, Kombinationsmöglichkeiten bzw. Bedeutungserklärungen enthalten und in manchen Fällen auch die phonetische Umschrift anbieten. Auf ein analoges Wörterbuch für Russischlernende warten wir schon seit Jahren!

Bei der Übersetzung von Fachtexten, in denen viele Fremdwörter vorkommen, die auf lateinischen und griechischen Stammorphemen aufbauen, ist es hilfreich, auch ein Wörterbuch der "falschen Freunde“ zur Hand zu haben, z.B. „Sprachfallen im Russischen“ von K.H.M. Gottlieb (Hueber-Verlag 1995). Man bekommt ab und zu Übersetzungen ins Deutsche oder ins Russische zu lesen, in denen die so bekannten Wörter wie intelligent, Motiv, Ökonomie, Maschine, Abiturient in eine der beiden Sprachen falsch übersetzt worden sind. Aber auch die aus dem Deutschen ins Russische entlehnten Wörter wiе концертмейстер, ландиафт, кронитейн, галстук haben im Russischen im Laufe der Zeit eine ganz andere Bedeutungsentwicklung genommen.

Für einen nächsten Arbeitsschritt benötigt man noch Parallel- und Hintergrundtexte im Russischen, um die Terminologieliste fuir das Russische zu erarbeiten. Erst dann können Begriffe und Benennungen in beiden Spra- 
chen zugeordnet und eine entsprechende Datenbank angelegt werden. Das Internet eröffnet hier neue Möglichkeiten: Mit Hilfe der Suchmaschinen kann man auf schnellem Wege zu bestimmten Fachtexten und Datenbanken gelangen.

An der Erstellung entsprechender multilingualer Datenbanken wird zunehmend gearbeitet. So entwickelt die Hochschule für Technik (FH) Karlsruhe seit 1997 im Rahmen eines Projektes eine Terminologiedatenbank TermIng (Terminologie im Ingenieurwesen) auf der Basis des Terminologieverwaltungsprogramms MuliTerm '95 Plus. Die Datenbank Termlng enthält drei Arten von Einträgen:

- terminographische Informationen, die dem Verständnis und der korrekten Verwendung von mutter- und fremdsprachigen Fachausdrücken dienen,

- textographische Informationen, die die Produktion von fremdsprachigen Texten entsprechend der jeweiligen Textsortenkonventionen unterstuitzen, und

- Informationen zu Kulturspezifika, die benötigt werden, um die Anpassungen im nonverbalen Bereich (z.B. unterschiedliche Darstellungskonventionen bei technischen Zeichnungen) vorzunehmen.

Neben Englisch, Französisch und Deutsch sind als mögliche Ausgangs- und Zielsprachen derzeit Spanisch, Portugiesisch, Italienisch und Russisch vorgesehen. Eine ausführliche Darstellung der Konzeption von TermIng findet sich bei Göpferich (1999). So eine Datenbank kann sicherlich den Recherchenaufwand erheblich verringern und zu einer höheren Qualität der Übersetzung von technischen und naturwissenschaftlichen Texten beitragen.

\section{2. Übersetzer und Fachwelt}

Bevor der Text in der Zielsprache verfasst wird, muss selbstverständlich mit dem Auftraggeber geklärt sein, welchem Zweck die Übersetzung dient, für welches Medium zu uibersetzen ist und welcher Bearbeitungsgrad des Zieltextes erwartet wird (z.B. Rohiibersetzung, Resümee oder druckreife Übersetzung).

Bei der Translation eines Fachtextes ist es besonders wichtig, von vornherein zu wissen, welche kommunikative Funktion der Zieltext erfuillen soll, ob er fuir fachinterne oder fachexterne Kommunikation bestimmt ist. Zu der Fachkompetenz eines Sprachmittlers gehört selbstverständlich auch das Wissen über die eventuellen Unterschiede bei der Zuordnung des jeweiligen Textes mit all seinen stilistischen Eigenschaften und kommunikativen Konventionen zu einer bestimmten Textsorte in der Ausgangs- und Zielsprache.

\subsection{Begriff Textsorte}

Zunächst einiges zum Begriff Textsorte. Aus der Analyse des Wortes selbst können wir schon verstehen, dass es sich hierbei um Klassen von Texten bzw. Mengen von Texten mit bestimmten Eigenschaften handelt. In der Literatur zur Textsortenproblematik finden sich je nach terminologischer Gewohnheit oder speziell definiertem
Verständnis auch andere Bezeichnungen, z.B. Texttypen, Textarten oder Gattungen. Ohne großes theoretisches Vorwissen klassifizieren wir in unserem Alltag intuitiv die Texte nach ihren Erscheinungsformen, z.B. Brief, Wetterbericht, Stellenausschreibung, Predigt oder eben Gebrauchsanweisung. Ebenfalls unterscheiden wir vortheoretisch die Texte nach Bereichen, z.B. literarische, juristische, wissenschaftliche, religiöse usw. (vgl. Lewandowski, 1990:1172ff.).

Mit der Texttypologie beschäftigt sich die Textlinguistik bereits seit ca. 30 Jahren. Der Differenzierung von Texten wurden unterschiedliche textinterne und textexterne Kriterien zugrunde gelegt. Bei den textinternen Kriterien geht es um textgrammatische und syntaktisch-semantische Aspekte, die in erster Linie mit der Sprachsystemspezifik zusammenhängen. Bei textexternen Kriterien handelt es sich um kommunikativ-pragmatische Eigenschaften bzw. Funktionen der Texte, die eher die Darstellungskonventionen, also die Kulturspezifik berïcksichtigen.

Ich werde mich hier in die Textsortenproblematik nicht weiter vertiefen und fuihre nur eine Definition des Begriffes Textsorte von K. Brinker (1985:124) an:

„Textsorten sind konventionell geltende Muster für komplexe sprachliche Handlungen und lassen sich als jeweils typische Verbindungen von kontextuellen (situativen), kommunikativ-funktionalen und strukturellen (grammatischen und thematischen) Merkmalen beschreiben. Sie haben sich in der Sprachgemeinschaft historisch entwikkelt und gehören zum Alltagswissen der Sprachteilhaber; sie besitzen zwar eine normierende Wirkung, erleichtern aber zugleich den kommunikativen Umgang, indem sie den Kommunizierenden mehr oder weniger feste Orientierungen für die Produktion und Rezeption von Texten geben."

Und noch eine wichtige Ergänzung von L. Hoffmann (1990:11) zum Begriff Fachtextsorte:

„Fachtextsorten sind eine spezielle Klasse von Textsorten, bei deren Produktion und Rezeption zusätzlich zum Alltagswissen noch Fachwissen nötig ist."

(Weiterfuihrende Literatur zu dieser Problematik s. Göpferich, 1998a).

Den Übersetzern geht es ja um die Produktion einer bestimmten Textsorte, die in der Zielkultur womöglich ganz anderen Konventionen unterliegt.

\subsection{Textsorte Bedienungsanleitung im Vergleich}

Aus der in der Vorbemerkung bereits genannten Untersuchung zur Marktsituation der Übersetzer von P. Schmitt (1988) lässt sich weiterhin entnehmen, dass die Benutzerinformationen, also Anleitungen, Produktdokumentation und Anweisungen jeglicher Art in der translatorischen Praxis einen herausragenden Platz einnehmen. Es ist deshalb ratsam, sich mit der Textsorte zu befassen und sich im Vorfeld kundig zu machen, was von einer Bedienungsanleitung im Land der Zielsprache erwartet wird. Die russischen Bedienungsanleitungen müssen nach dem sowjetischen Standard GOST 26119-84 aus dem Jahre 1984 verfasst werden, der den Bestand, die Abfolge und den Status der Teiltexte exakt festlegt ${ }^{6}$. 
Bei einer näheren Betrachtung einiger russischen Bedienungsanleitungen konnte folgendes festgestellt werden: Die Benutzerinformationen für Waren, die in Russland hergestellt wurden, halten im großen und ganzen die GOST-Normen ein. Die Waren, die importiert werden, führen die Bedienungsanleitungen in Russisch mit, die fast komplett den westlichen Konventionen folgen. Hier ist sicherlich eine gewisse Textsortendynamik eingetreten, da sowohl in bezug auf sprachliche Darstellungsweise als auch auf Informationsanordnung einige Veränderungen festzustellen sind.

Neu ist in den ins Russische übersetzten Bedienungsanleitungen folgendes:

- die appellative Komponente, d.h. Annäherung an die Textsorte Werbung. Es sind vor allem Gluickwuinsche zum Kauf des richtigen Gerätes, etwas Werbung für das Produkt u.ä..

- Weiterhin ist zu beobachten, dass die sprachlichen Formulierungen viel kundenfreundlicher, schlicht verständlicher geworden sind.

- Als Einfluss der westlichen orthographischen Normen verstehe ich die Großschreibung des Personalpronomens Вы als Ausdruck eines höflichen Umgangs mit dem Kunden. Das russische orthographische Regelwerk schreibt es nämlich nicht vor:

- Auffällig ist auch die Nicht-Übersetzung einiger Bezeichnungen, genauer gesagt, die ausbleibende Überführung in die kyrillische Schrift, z.B. Haжsмume клавишу „Enter“, Standby-Modus, „карточка SIM“ oder Kинескоn „Diamondtron“ u.ä..

- Es werden zuweilen im Prinzip überflüssige Informationen uibersetzt, die auch hierzulande eher die Funktion der „Politischen Korrektheit“ erfüllen, z.B. „Umweltschutz ist auch unsere Aufgabe. Dieses Handbuch wurde von der ersten bis zur letzten Seite auf Papier gedruckt, bei dessen Herstellung keinerlei Chlor mehr verwendet wird." 7

- Ebenfalls wenig Sinn für den russischen Verbraucher machen die Hinweise zur sachgerechten Entsorgung von Verpackung und Altgeräten, weil das entsprechende Entsorgungssystem in der hier bekannten Form dort nicht vorhanden ist. Aber da diese Informationen nicht negativ bewertet, eher mit Schmunzeln registriert werden, stören sie die Funktion des Textes nicht. (Allerdings habe ich nie feststellen können, dass der Satz „Der Händler ist verpflichtet, die Verpackung zurückzunehmen" übersetzt worden wäre.)

\section{3. Übersetzer und technisches Gerät}

\subsection{Das „Handy“}

Im folgenden will ich auf die Übersetzung einer Bedienungsanleitung für ein Funktelefon der Firma Siemens aus dem Deutschen ins Russische eingehen, ein Gerät, das im Volksmund liebevoll mit dem pseudoenglischen Wort „Handy“ bezeichnet wird. (Wir kennen viele solcher neu-deutscher Wortschöpfungen wie z.B. Fineliner, Hairshop, u.a.).
Die Nachfrage nach Funktelefonen kam mit der „Handy-Welle“ auch nach Russland. Für einen „Neuen Russen“ ist das kleine Gerät allein aus Statusgrüinden ein absolutes Muss. Hier ließe sich die oft zitierte, berühmte Passage "И какой же русский не любит быстрой езды ...?» (wörtlich: Welcher Russe fährt schon nicht gern schnell ...?) aus Gogols „Mёртвые души“ paraphrasieren, genauer modernisieren: " $h$ какой же новый русский не любит сотового телефона ....» (= Welcher Russe mag schon nicht das Funktelefon?).

Die Funktelefone der Firma Siemens finden auf dem russischen Markt einen guten Absatz. Das Gerät wird selbstverständlich mit entsprechenden Gebrauchsanweisungen in russischer Sprache verkauft. Das Interesse an einem solchen Textpaar (Ausgangs- und Zieltext) aus der translatorischen Perspektive liegt darin begründet, dass sich hier die Anpassung der Benutzerinformationen an den kulturell anders geprägten Verbraucher gut beobachten lässt.

Der zu besprechende Text „Benutzerhandbuch“ ist eine Gebrauchsinformation und gehört zum Bereich Fachtext Technik, Textsorte Anweisung, Bedienungsanleitung. Der Text ist für die fachexterne Kommunikation bestimmt. In bezug auf diese Textsorte gilt es, die Kommunikation zwischen dem Ausgangstextsender und dem Zieltextempfänger so herzustellen, so dass der letztere nicht bemerkt, dass es sich hierbei um eine Übersetzung handelt. Nord (1993:25) spricht hier vom „instrumentellen" Übersetzungstyp im Unterschied zum „dokumentarischen“ Typ.

Bei der Übersetzung der Textsorte Bedienungsanleitung erübrigt sich die manchmal recht qualvolle Suche nach einem angemessenen Titel für den Text, denn die Bezeichnung der entsprechenden Textsorte im Russischen ist vorhanden. Wir übersetzen also den deutschen Texttitel „Benutzerhandbuch" ganz konventionell als „Инструкция по обслуживанию ....”.

Die Benennung der Ware „Funktelefon“ muss in der Zielsprache auch nicht neu erfunden werden, da sie bereits im Russischen existiert. Das Gerät bzw. die Ware selbst trägt im Russischen die Bezeichnung „сотовый телефон“. Das bedeutet im Russischen soviel wie „Funknetz in Form von Waben“. Im Umlauf gibt es noch ein paar Bezeichnungen wie z.B. „радиотелефон“, „мобильный телефон“, oder einfach „сотовый“ und im Jugendjargon „мобильник“.

In unserem Text entfällt weiterhin das Problem der Übersetzung von Eigennamen. Sie ist nur dann erforderlich, wenn aufgrund der Lautkombination unerwünschte Assoziationen entstehen. So könnte man z.B. das russische Parfüm „Миф“ unter diesem Namen im deutschsprachigen Raum nicht verkaufen. Die Übersetzung als „Mythos“ bzw. eine Umbenennung wäre hier geboten. Bei der Übersetzung einer Bedienungsanleitung werden wir auch nicht mit dem Problem der Übertragung von Phraseologismen konfrontiert. 
Die Nutzung des Funktelefons erfordert vom Verbraucher zwar keine spezielle technische Vorbildung, setzt dennoch etwas Erfahrung im Umgang mit digitaler Technik voraus. Der Vergleich der sich bereits im Umlauf befindenden russischen Übersetzung mit dem Originaltext wirft einige „technische“ Fragen auf, die im nächsten Abschnitt genannt werden.

\subsection{Technischer Fachtext und seine „technischen“ Probleme}

Aus der Gegenüberstellung einiger Passagen des Originaltextes und entsprechenden Stellen der russischen Übersetzung ergeben sich einige Differenzen in der Informationswiedergabe.

Die Einleitung beginnt mit der (im Russischen früher unüblichen) Begrïßung des neuen Kunden.

Willkommen im PCN-Netz

(Personal Communication Network)!

Добро пожаловать в сеть мобильной радиосвязи РСN!

Wie oben bereits erwähnt, werden einige Bezeichnungen und erst recht die Abkürzungen nicht in die kyrillische Schrift uiberfuihrt. Die Abbreviatur PCN wird in der russischen Übersetzung allerdings erklärt (сеть мобильной радиосвязи), aber neben dem russischen Textsegment im Unterschied zum deutschen Ausgangstext beibehalten, wo Englisch wie selbstverständlich stehen bleibt. Die Abkuirzung PCN fungiert offensichtlich als eine Art Bezeichnung des Netztyps und somit ist es auch im Deutschen (PCN-Netz) nicht als Pleonasmus zu verstehen.

Der nächste Absatz mit der Information über den technischen Standard des Netzes (,Ihr Telefon entspricht der DCS-1800-Digitalnorm...") wird komplett in der russischen Übersetzung ausgelassen. Es ist durchaus möglich, dass die russischen digitalen Kommunikationssysteme auf anderen technischen Standards basieren. Dem russischen Kunden wird eine entsprechende Information jedoch vorenthalten.

Es wird hier nicht beabsichtigt, alle 50 Seiten des Ausgangs- und Zieltextes durchzugehen und mögliche Übersetzungsvarianten zu besprechen. Dies wäre auch eine Arbeit, die uns zu weit auf die mikrostrukturelle Ebene führen und den Rahmen dieses Aufsatzes sprengen würde. Hier soll nur noch ein „technisches“ Problem angesprochen werden, das in dieser Translation vorgekommen ist.

In dem Abschnitt Textnachrichten_wird dem Telefonnutzer erklärt, wie man Nachrichten sendet, liest und löscht. Der Text auf dem Display ist fuir den russischsprachigen Benutzer durchgehend in Russisch (z.B. меню, выбор) verfasst. Das Problem liegt für den russischsprachigen Nutzer allerdings darin, dass der eigene Nachrichtentext nicht ohne weiteres in Kyrillisch geschrieben werden kann, da die numerische Tastatur mit lateinischem Alphabet belegt ist. In der russischen Übersetzung stehen die Textnachrichten - ohne jede Erklärung! - in Englisch, z.B.: 1-я строка сообщения:

«arrival 4 pm»

Последняя строка сообщения:

«can your confirm? Regard Jane»

An keiner Stelle der russischen Bedienungsanleitung finden wir einen Hinweis, wie der russische Kunde mit diesem Problem fertig werden soll. Ich habe diesen merkwürdigen Umstand mit ein paar russischen Studenten und mit anderen Bekannten, die in Russland leben, besprochen. Überraschenderweise hat sich herausgestellt, dass es die Besitzer von Funktelefonen überhaupt nicht stört, dass die Textnachrichten nicht geschrieben werden können! Mit diesem - aus meiner Sicht - technischen Mangel gehen die russischen Nutzer sehr gelassen um. Die von mir befragten Personen nehmen diese Funktion des Funkgerätes ïberhaupt nicht in Anspruch. Einige meinten, man kann bei Bedarf die russischen Wörter auch in lateinischen Schrift schreiben, z.B. „Priletaju sawtra...” (= komme morgen). Ein Student berichtete allerdings, dass es ihm nach längerem Ausprobieren - „Методом научного тыка“ (= Handeln nach dem „wissenschaftlichen" Zufallsprinzip), wie er sich ausdrüickte, - doch noch gelungen ist, eine Textnachricht in Kyrillisch zu schreiben. Er konnte aber die Schritte nicht mehr rekonstruieren.

\section{3. Übersetzer und verschiedene "technische“ Probleme}

Der besprochene Abschnitt der übersetzten Bedienungsanleitung macht deutlich, dass die Anpassung des Textes bzw. des Gerätes an den russischsprachigen Verbraucher nicht optimal erfolgte. Die Benutzung des Funktelefons setzt die Beherrschung der lateinischen Schrift, der englischen Sprache sowie ein Stiick „Pfiffigkeit“ voraus.

Die Hersteller - hier Siemens - halten es offensichtlich nicht für erforderlich, hier noch zu investieren, da der Umsatz der Funktelefone auf dem russischen Markt davon höchstwahrscheinlich nicht abhängig ist. Möglicherweise verfügen die Funktelefon-Nutzer (auch die „Neuen Russen“) über genügend Englisch-Kenntnisse, so dass hier keine Kommunikationsstörung eintritt.

Hier erhebt sich aber eine Reihe von Fragen:

- Ist der genannte Sachverhalt uiberhaupt ein Problem der Übersetzung oder des Übersetzers?

- Welche Einflussmöglichkeiten hat ein Übersetzer beim Auftraggeber?

- Wo hört die Verantwortung des Übersetzers auf?

- Es ist weiterhin auch eine nicht immer eindeutig zu beantwortende Frage, ob eine Entscheidung - Abschnitte auszulassen und Informationen hinzuzufuigen - überhaupt in der Kompetenz des Übersetzers liegt. Handelt es sich hierbei um eine Art kulturelle Anpassung des Textes auf Kosten des Informationsumfangs?

- Ist dies noch im Sinne des Verbrauchers?

Auf diese Fragen kann es sicherlich keine situationsunabhängigen Antworten geben. Es ging hier darum, 
einige Probleme nicht sprachlicher Art aufzuzeigen, mit denen der Sprachmittler bei der Übersetzung eines Textes konfrontiert wird.

[1] Ich erlaube mir, hier eine okkasionelle Deutung des Begriffes „Internationalisierung" vorzunehmen, dessen inhaltliche Bestimmung im öffentlichen Diskurs bedauerlicherweise genau so wenig erfolgt wie die des ebenfalls sehr beliebten Begriffes „Globalisierung“.

[2] Aufsatz von J.Schmadl/ J-U. Gerking, in: Wissenschaftliche Beiträge der TFH Wildau, 1998/2, S. 106-110.

[3] Wissenschaftliche Beiträge der TFH Wildau, 1996/1, S. 39.

[4] Unter Paralleltexten werden in der Fachliteratur verschiedensprachige Texte verstanden, „die originär in ihrer jeweiligen Sprache - am besten von kompetenten Muttersprachlern - erstellt wurden, die also keine Übersetzungen voneinander sind, aber ein möglichst ähnliches Thema behandeln und sich in ihrer kommunikativen Funktion entsprechen, d.h. derselben Textsorte (nvariante) angehören“. (vgl. Göpferich, 1998b:184).

[5] Unter Hintergrundtexten versteht man Texte unterschiedlicher Sorte zu gleicher Thematik.

[6] Zitiert nach E. Fleischmann (1999).

[7] Belinea, Bedienungsanleitung zum Monitor

\section{Literatur}

\section{Fachliteratur}

[1] Brinker, Klaus (1985): Linguistische Texanalyse. Eine Einfuihrung in ihre Grundbegriffe und Methoden, Berlin.

[2] Fleischmann, Eberhard (1999): „Zum Problem der Kulturspezifik in der fachlichen Kommunikation“, in: Rösch, O.: "Interkulturelle Kommunikation mit polnischen Partnern in Wirtschaft und Wissenschaft", Wildauer Schriftenreihe Interkuklturelle Kommunikation Band 3, 1999, S. 135-156.

[3] Göpferich, Susanne (1998a): „Text, Textsorte, Texttyp“, In: Snell-Honby, M. et al. (Hrsg.) Handbuch Translation. Verlag Stauffenburg Tübingen, 1998, S. 61-64.

[4] Göpferich, Susanne (1998b): „Paralleltexte“, In: SnellHonby, M. et al. (Hrsg.) Handbuch Translation. Verlag Stauffenburg Tübingen, 1998, S. 184-186.

[5] Göpferich, Susanne (1999): „Kulturspezifik im Umfeld von Terminologie: Informationsangebot und Struktur von TermIng, der Terminologiedatenbank der Hochschule fuir Technik (FH) Karlsruhe“, in: Satzger, A. „Sprache und Technik“, Forum Angewandte Linguistik, Bd. 36, 1999, S. 85120.

[6] Hoffmann, Lothar (1990): „Fachtexte und Fachtextsorten“, Leipzig 1990 (BSF Berichte der Sektion Fremdsprachen 5).

[7] Hönig, Hans (1997): „Konstruktives Übersetzen. Studien zur Translation", Bd. 1, 1997, Tübingen, Verlag Stauffenburg, 195 Seiten.

[8] Nord, Christine (1993): „Einführung in das funktionale Übersetzen am Beispiel von Titeln und Überschriften“, 1993 A. Franke-Verlag Tübingen und Basel, 312 Seiten.

[9] Schmitt, Peter A. (1998): „Marktsituation der Übersetzer“, In: Snell-Honby, M. et al. (Hrsg.) Handbuch Translation. Verlag Stauffenburg Tuibingen, 1998, S. 5-13.

\section{Nachschlagewerke}

[10] Birkenmaer, Willi /Mohl, Irene (1990): „Bibliographie zur russischen Fachsprache. Forum für Fachsprachenforschung“, 1990, Tübingen.

[11] Fachwörterbuch Mikroelektronik (1991): Englisch Russisch Deutsch Französisch Niederländisch, Klett, Kluwer, 1991.

[12] Gottlieb, K.H.M. (1985) „Sprachfallen im Russischen. Wörterbuch der, falschen Freunde' Deutsch und Russisch. Ein Lehr- und Nachschlagewerk". Max Hueber Verlag, 1985, $159 \mathrm{~S}$.

[13] Langenscheidts Großwörterbuch Deutsch als Fremdsprache. Das neue einsprachige Wörterbuch für Deutschlernende (1997) Langenscheid, Berlin/ München/ Wien/ Zürich/ New York.

[14] Lewandowski, Theodor; (1990), Linguistisches Wörterbuch in 3 Bänden, Quelle\&Meyer, Heidelberg/ Wiesbaden, 1990

[15] Marktwirtschaft und Planwirtschaft. Ein enzyklopädisches Wörterbuch, (1992), Deutsch-Englisch-Russisch, 2 Bde, K.G. Saur, München/London/ New York/ Paris, 1992.

[16] Polytechnisches Wörterbuch (1995), Deutsch-Russisch, A. Pankin, Moskau 1995

[17] Slowar" sotschetajemosti slow russkogo jasyka (1983) Словарь сочетаемости слов русского языка под редакцией Денисова П.Н. и Морковина В.В., Москва «Русский язык» 1983, издание 2-е, исправленное.

[18] Wörterbuch Wirtschaftsrussisch: Schule für das internationale Business. (1993), Deutsch-Russisch, Anna S. Nikiforowa. Cornelsen Verlag Berlin.

\section{sonstige Quellen}

[19] Belinea 107050 User Manual in Twelve Languages (1997), Bedienungsanleitung zum Monitor, Copyright M1997, MAXDATA, Marl.

[20] Benutzerhandbuch Telefon DCS 1800, S6 PCN (in Deutsch und Russisch)

[21] Lehrmaterialien „Qualitätsmanagement“ (unveröffentlichte Arbeitsmaterialien)

[22] Schmadl, Josef / Jens-Uwe. Gerking (1998): „Stufenwirkungsgrad und Extraktionsmittelverbrauch in der FlüssigExtraktion: Vergleich Mittler-Settler und pulsierter Siebboden in Miniplanttechnik“, in: Wissenschaftliche Beiträge der TFH Wildau, 1998/2, S. 106-110.

\section{Autorin}

\section{Dr. phil. Olga Rösch}

Technische Fachhochschule Wildau

Leiterin des Sprachenzentrums und des

Instituts für Interkulturelle Kommunikation

Bahnhofstraße

15745 Wildau

Tel. +49 (0) 3375 - 508-367 und 508-348

E-Mail: roesch@sprz.tfh-wildau.de 